\title{
ANNA - die Ärztin mit der spitzen Tintenfeder
}

\author{
Ähnlich wie ein Chirurg setzt auch sie ihre manuellen Fähigkeiten ein und seziert auf \\ ihre eigene Art: Mit Tintenfeder und Papier karikiert ANNA aktuelle Geschehnisse im \\ Gesundheitsbereich. Ihre Karikaturen sind seit 10 Jahren fester Bestandteil in der \\ SÄZ. Ein Besuch bei Frau Doktor mit der spitzen Feder.
}

«Wer ich bin?», fragt ANNA. Sie lacht schelmisch. «Ich bin eigentlich Medizinerin, doch habe ich mich dazu entschieden, das zu machen, was ich wirklich kann.» Und das ist Karikieren. Ärzte, Patienten und Politiker vor keinem macht ihre spitze Feder halt. Auch nicht vor sich selbst. Ihre Selbstkarikatur erscheint in der SÄZ jeweils zusammen mit ihrer aktuellen Karikatur. Ihr Markenzeichen: ultrakurze Haare, ein strenger Blick durch die Brille und ein verschmitztes Lächeln. Doch eigentlich sieht sie sich selbst nicht gerne als Karikatur: «Ich selbst bin sehr empfindlich, vor allem, wenn ich mich auf der Darstellung nicht mag», gesteht ANNA, «Fotos, die mir nicht passen, kann ich zerreissen, aber nicht eine Karikatur.»

Weit über 3000 Karikaturen hat ANNA - alias Anna Regula Hartmann-Allgöwer - schon gezeichnet. Und doch spürt die Künstlerin das Heimweh nach der Medizin: «Ich wäre auch gerne Ärztin geworden. Sagen wir es so: Ich hätte auch mit manchem anderen Mann

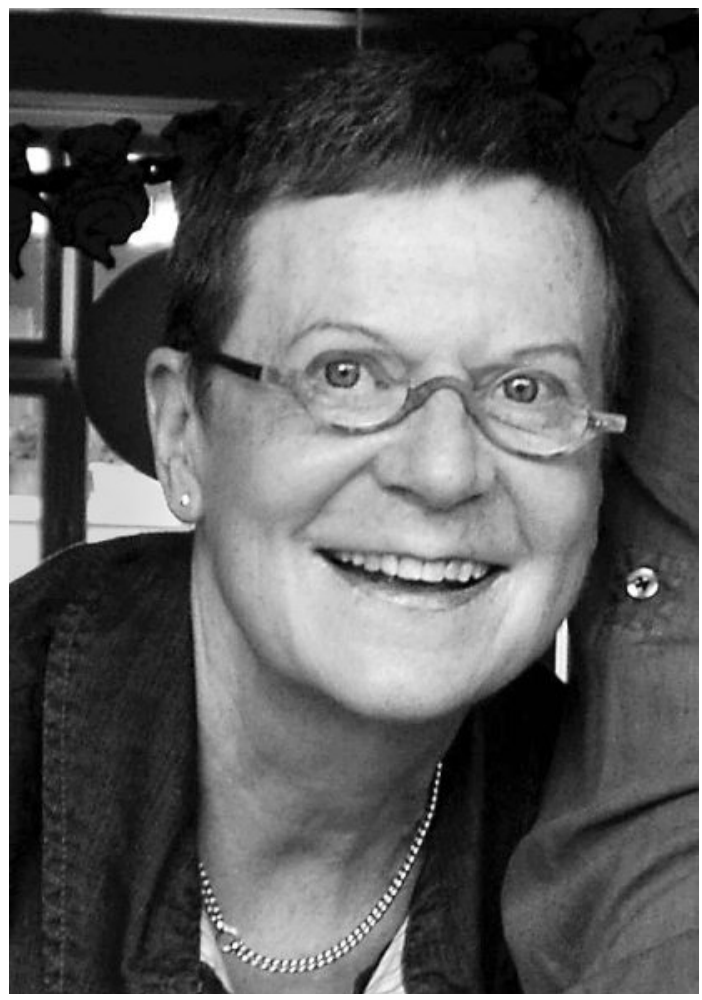

\begin{abstract}
ANNA - depuis 10 ans dans le BMS
A l'instar d'un chirurgien, elle utilise ses compétences de dissection pour œuvrer non pas au scalpel mais à la plume. Avec de l'encre et du papier, ANNA caricature pour croquer l'actualité du domaine de la santé. Ses dessins sont incisifs et en font apparaître plus d'un sous son vrai visage. Avec volupté et tendresse, elle débusque I'humain dans ses faiblesses si terriblement humaines, et touche donc aussi les médecins. «Avec mes caricatures, je veux soutenir les médecins dans leur autocritique et bien que je montre souvent le côté négatif, c'est le côté positif que j'entends souligner» explique-t-elle. En montrant le côté sans cœur et injuste de notre société, la dessinatrice plaide pour plus d'attention et de gentillesse et couche sur le papier ce qui lui brûle l'âme.
\end{abstract}

eine gute Ehe führen können, trotzdem habe ich das Gefühl, den richtigen ausgesucht zu haben.» Immerhin ist sie für ihre Familie und Freunde noch immer die «Barfuss-Ärztin», die bei kleineren Problemen jeglicher Art weiterhelfen kann.

\section{ANNA entlarvt die menschlichen Schwächen}

Ihre Karikaturen sind bissig und sie zeigen manchen mit seinem wahren Gesicht. Lustvoll und liebevoll zugleich entlarvt ANNA den Menschen und seine allzu menschlichen Schwächen. Und dies gilt auch für die Ärzte. «Mit den Karikaturen möchte ich sie in ihrer Selbstreflexion unterstützen, dabei stelle ich oft das Negative dar, möchte aber damit das Positive provozieren», erklärt ANNA. Ihre Zeichnungen thematisieren Lieblosigkeiten und Ungerechtigkeiten in unserer Gesellschaft, doch möchte ANNA damit Achtung und liebevolle Zuwendung erreichen: «Ob ich dies wirklich erreiche, weiss ich nicht. Aber zumindest kann ich darauf hinweisen - eigentlich ein langweiliger moralischer Anspruch.» Doch was ihr auf der Seele brennt, bringt sie auf Papier.

Ein Dauerbrenner ihrer Themen: die Altersdiskriminierung. «Vor allem alte Frauen werden diskriminiert. Welcher Arzt hat sich nicht schon dabei ertappt, wie er vor der Behandlung heimlich einen Blick auf die 
Patientenkarte geworfen hat, und bei Jahrgang 23 die Nase gerümpft, sich hingegen bei Jahrgang 85 auf eine junge Patientin gefreut hat. Aber das ist menschlich!», tröstet ANNA. Reaktionen von Lesern erhält sie meistens dann, wenn sie die Themen Religion und Sexualität thematisiert - und kommentiert. Mails von erzürnten Lesern füllen ihren Posteingang, wenn sie sich respektlos gegenüber dem Arztberuf gibt.

Auch im Wartezimmer macht sie sich ein Bild ANNA ergreift jede Chance, sich neue Ideen für Karikaturen zu holen. Da sie selbst nicht praktizierende Ärztin ist, macht sie sich auf zwei Arten ein Bild über die heutigen Ärzte: einerseits im Freundeskreis, hier hört sie sich Geschichten und Meinungen an, andererseits greift sie alle interessanten Kontakte auf, die sich

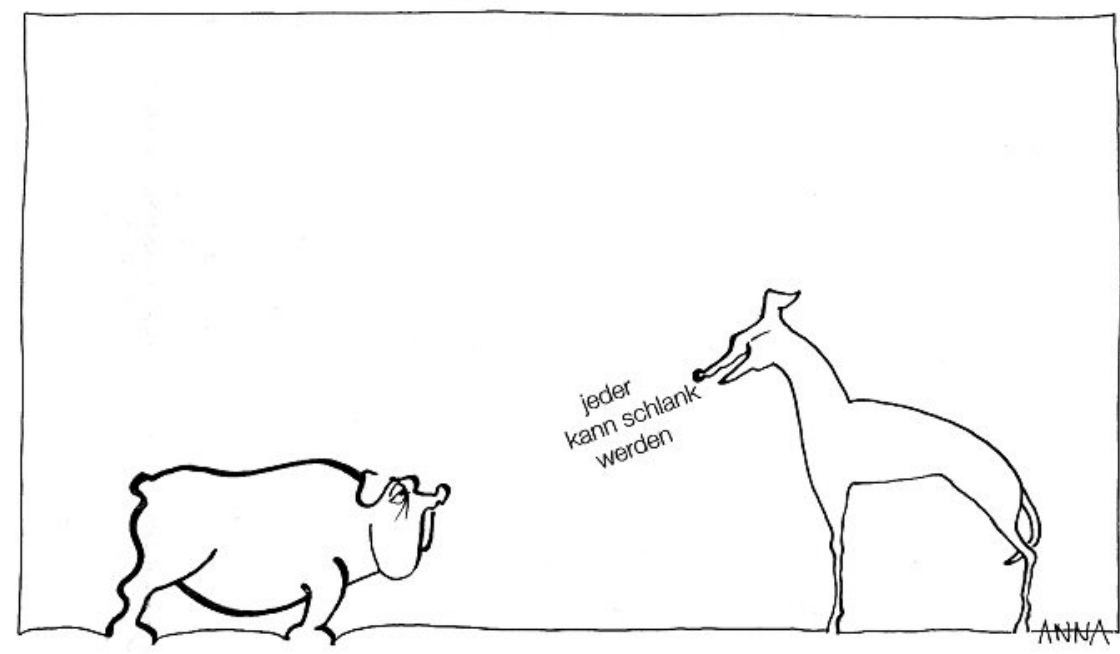

aus Alltagssituationen heraus ergeben. «Dass ich eine gewisse Distanz zum Ärztealltag habe, hat sich bisher nicht negativ ausgewirkt», meint sie, «ich bin die Beobachterin des Geschehens.» Ist ANNA auf Spitalbesuch, schaut sie sich gerne im Gebäude um, oder auch in Wartezimmern. Das Beobachtete hält sie als Skizze in ihrem «Beobachtungsbüchlein» fest, das sie stets in ihrer Handtasche trägt. «Ich darf nicht faul werden. Die Welt verändert sich ständig. Krankenschwestern haben heute keine Häubchen und Ärzte keine Stirnspiegel mehr», meint ANNA und lacht, «das ist schade für mich!».

Doch ANNA nimmt längst nicht nur Ärzte ins Visier. Auch die Patienten bekommen ihr Fett ab: beispielsweise der Patient als Konsument. Sie zeichnet ihn als gierigen Konsumsüchtigen, der sich seinen Einkaufswagen bis an die Decke vollpackt und so seine Krankenkassenprämien wettmachen will. «Auch Patienten sollen merken, wie konsumorientiert sie in Bezug auf die Medizin handeln», erklärt ANNA, «diese Mentalität ist einfach verheerend».
«Meine Zeichnungen sind kalorienreduziert»

Auch die Überkonsumation von Alternativmedizin thematisiert ANNA gerne. An Themen mangelt es ihr nie: «Es gibt immer mehr Themen, als ich aufnehmen kann. Das Gesundheitswesen ist so vielseitig und umfasst viele menschliche Bedürfnisse.» Derzeit sind ihre Dauerbrenner die hohen Krankenkassenprämien und Medikamentenpreise, die Aufwertung der Hausarztmedizin sowie die Ressourcenverteilung in der Altersmedizin. Ein weiterer Schwerpunkt, der ANNA beschäftigt: Frauen und Teilzeitarbeit in der Medizin. «Ich finde es nicht fair, dass Frauen in Teilzeitarbeit nicht zugeben können, dass sie weniger Erfahrung haben als ein Mann, der zu 100 Prozent arbeitet. Das haben sie einfach nicht!».

Ihre Karikatur bezeichnet ANNA als «Konzentrat». Mit Reduktion auf das Minimum will sie das Maximum herausholen. Diese Reduzierung widerspiegelt sich nicht nur im Inhalt, sondern auch im Stil. Ihr Rezept: kein Punkt, kein Strich, keine Farbe zu viel. Die meisten ihrer Karikaturen bestehen nur aus einem einzigen Strich, der durchgehend durch das Bild führt. «Meine Zeichnungen sind kalorienreduziert», erläutert die Karikaturistin, «es ist wie 100 Liter Fleischsuppe zu einem kleinen Maggiwürfel einzukochen.»

\section{Karikieren ist «Gehirnjogging»}

Karikieren ist nicht nur ANNAS Arbeit und Leidenschaft, sondern auch ihr Fitnesstraining: «Es ist mein Gehirnjogging.» Mit der Präzision eines Chirurgen zeichnet sie so lange, bis sie einen einzigen Gedanken herausgearbeitet hat. Manchmal setzt sie ihre Tintenfeder 60 Mal für dieselbe Zeichnung an - so lange, bis sie inhaltlich und formal zufrieden ist.

Wenn ANNA nicht für die Schweizerische Ärztezeitung arbeitet, zeichnet sie im Auftrag Porträts oder Illustrationen für private Kunden für Bücher. Zurzeit illustriert sie ein Geriatrie-Buch für Hausärzte und eines über vorgeburtliche Untersuchungen bei Schwangeren. «Das Medizinstudium hilft mir bei meiner Arbeit sehr viel. Ich habe die Illusion, dass ich den Menschen so besser kenne», schildert ANNA, «aber ich war auch enttäuscht vom Medizinstudium. Ich hatte mehr erwartet, denn ich wollte alles lernen über Leben, Sterben und Gebären.»

Wenn ANNA nicht an ihrem Schreibtisch im Atelier sitzt und zeichnet, dann ist sie «Omanna», die Enkel hütende ANNA. «Das ist auch ein Job», meint sie, «aber ein wunderschöner.» Zeit fürs Karikieren bleibt weniger, deshalb macht sich ANNA Gedanken darüber, sich langsam von der SÄZ zurückzuziehen zeichnen aber wird sie lebenslänglich

Feedbacks zu ihren Arbeiten sind ANNA immer willkommen. Sie ist unter annah@rtmann.ch per E-Mail erreichbar. 